\title{
The behaviour of mosquitoes in relation to humans under holed bednets: the evidence from experimental huts
}

\author{
Seth R Irish/ ${ }^{+}$ \\ Entomology Branch, Department of Parasitic Diseases and Malaria, Center for Global Health, \\ Centers for Disease Control and Prevention, Atlanta, GA, USA
}

The physical integrity of bednets is a concern of national malaria control programs, as it is a key factor in determining the rate of replacement of bednets. It is largely assumed that increased numbers of holes will result in a loss of protection of sleepers from potentially infective bites. Experimental hut studies are valuable in understanding mosquito behaviour indoors, particularly as it relates to blood feeding and mortality. This review summarises findings from experimental hut studies, focusing on two issues: (i) the effect of different numbers or sizes of holes in bednets and (ii) feeding behaviour and mortality with holed nets as compared with unholed nets. As might be expected, increasing numbers and area of holes resulted in increased blood feeding by mosquitoes on sleepers. However, the presence of holes did not generally have a large effect on the mortality of mosquitoes. Successfully entering a holed mosquito net does not necessarily mean that mosquitoes spend less time in contact with the net, which could explain the lack in differences in mortality. Further behavioural studies are necessary to understand mosquito behaviour around nets and the importance of holed nets on malaria transmission.

Key words: mosquito net - holes - malaria - experimental hut - Anopheles - Culex

The distribution of pyrethroid-treated bednets is one of the primary activities of malaria control programs throughout the world and is also propounded as a tool for prevention of lymphatic filariasis and other mosquito-borne diseases (van den Berg et al. 2013). As there is currently only one class of insecticide that is safe and appropriate for net treatment (pyrethroids), the normal insecticide resistant management techniques, such as rotation or mixtures, are limited. Target site and metabolic resistance to pyrethroids have been widely reported (Ranson et al. 2011), however, the development of these resistance mechanisms does not necessarily result in a loss of effectiveness of insecticide-treated nets (Darriet et al. 2000, Henry et al. 2005, Corbel \& N'Guessan 2013). Nonetheless, reductions in blood feeding prevention and mortality rates have been noted in some situations (N'Guessan et al. 2007, Asidi et al. 2012). The entomological and epidemiological impact of this resistance is still under investigation (Ranson et al. 2011, WHO 2012, Strode et al. 2014).

Coupled with worries about developing resistance of mosquito vectors are concerns about the physical integrity of bednets, which may affect the protection that nets can provide (Rehman et al. 2011), as well as the use of the net (Batisso et al. 2012). Holes in nets are very common (Tami et al. 2004, Mejía et al. 2013) and, in some cases,

doi: 10.1590/0074-0276140159

Financial support: CDC as part of the President's Malaria Initiative

+ Corresponding author: sirish@cdc.gov

Received 9 May 2014

Accepted 15 August 2014 holes are found after fairly short periods of use (Kilian et al. 2011). Some steps have been made by net manufacturers to use stronger materials, such as polypropylene [eg., LifeNet (Bayer CropScience, France)] or to reinforce areas of nets where holes are likely to tear, [eg., PermaNet 3.0 (Vestergaard-Frandsen, Switzerland)], although some types of tears (due to animals or fires) would still damage these reinforced nets.

The evaluation of long lasting insecticidal nets (LLINs) occurs in three phases: laboratory testing, experimental hut trials and small scale field trials (WHO 2013). It is in Phase II experimental hut trials that the behaviour of mosquitoes can be most closely observed, through the blood feeding, mortality and location of collection of mosquitoes. Many studies have tried to estimate the effectiveness of used nets by cutting holes in nets, or bringing in nets that have been used in the field. These provide information on mosquito behaviour around holes with nets, particularly as it relates to blood feeding and mortality, the key parameters for disease transmission. Many important questions about the behaviour of mosquitoes around nets will have to be assessed experientially, but the aim of this review is to investigate the effect of holes in nets on mortality and blood feeding rates of mosquitoes reported from published experimental hut studies.

\section{MATERIALS AND METHODS}

A systematic literature search was conducted in September 2013. To find all articles describing experimental huts, the following databases were searched: PubMed (National Center for Biotechnology Information, National Library of Medicine, National Institutes of Health), the Armed Forces Pest Management Board Literature Retrieval System, IBECS, LILACS, Horizon Pleins Texts (database of the Institut de Recherche pour 


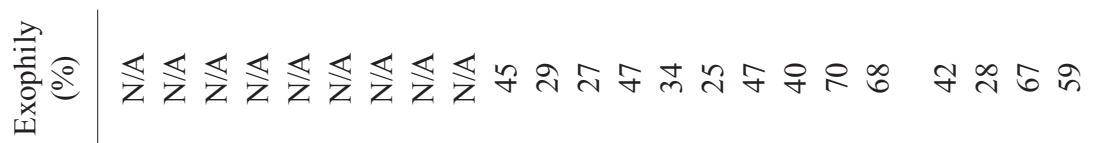

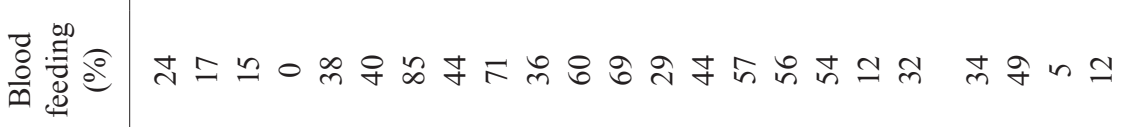

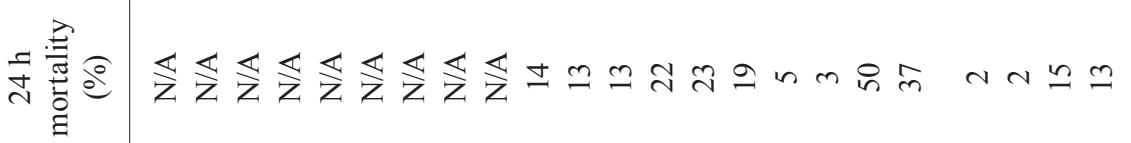

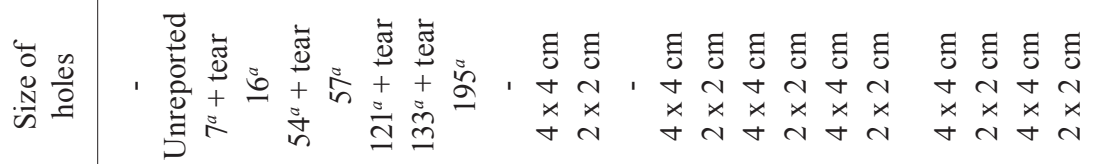

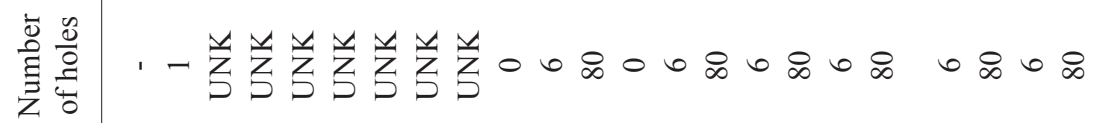

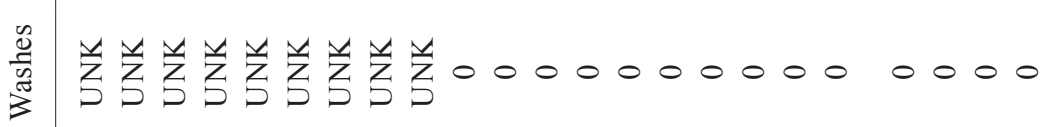

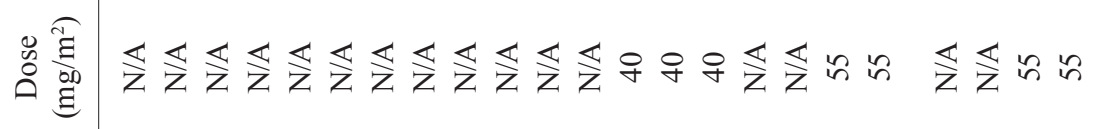

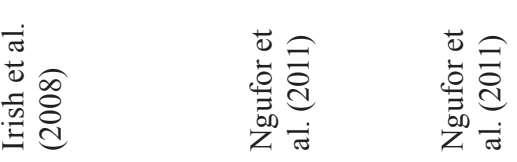




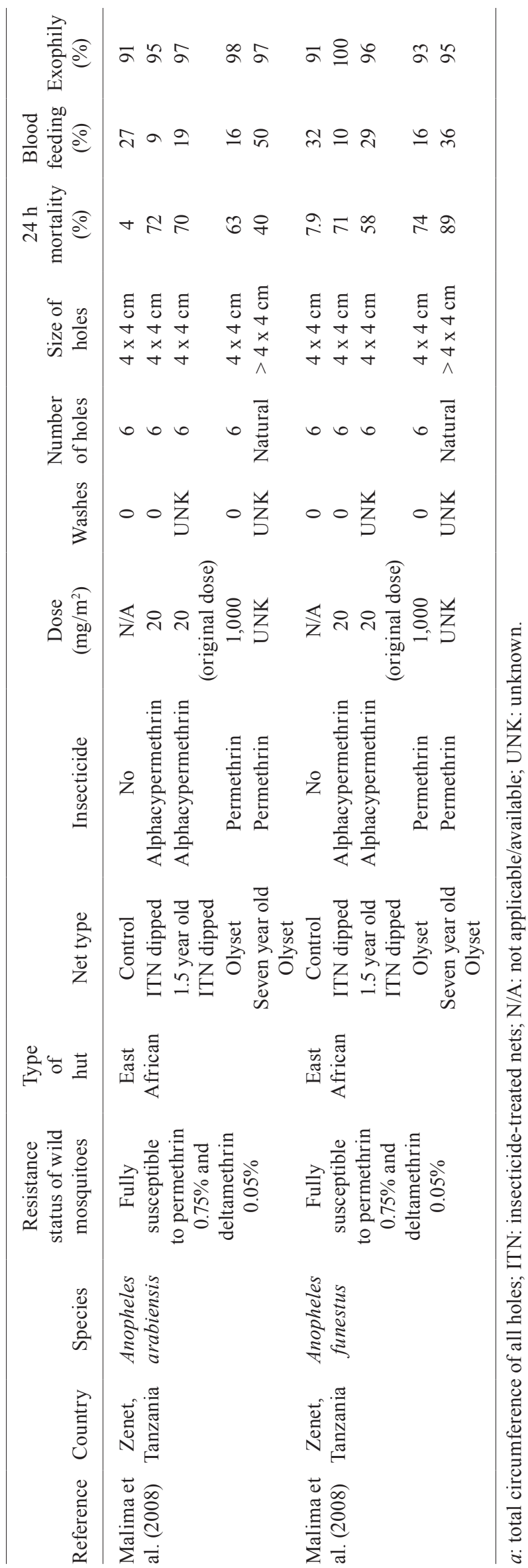

le Développement), the Web of Science, the Institutional Repository for Information Sharing of the World Health Organization (WHO) and the Cochrane Library. In addition, reference lists in the selected papers were screened to find additional articles. The following search terms were used: experimental hut, experimental huts, case piège, cases pièges, veranda hut, verandah huts, veranda hut, verandah huts, window trap hut, window trap huts, case expérimentale, cases expérimentales, veranda trap hut, veranda trap huts, verandah trap hut and verandah trap huts. Studies were selected if they met one of the following criteria: (i) an experimental hut study in which nets with different sizes or numbers of holes were used and (ii) an experimental hut study in which holed and unholed nets were used.

\section{RESULTS}

The search identified 1,051 articles of which 13 discussed experimental hut trials using holed bednets and met the criteria listed above (experiments described in report and article form or reported in two different languages were treated as one report). The results of these experiments are discussed within the appropriate theme below.

Studies with different size holes - Very few studies used nets with different sizes of holes. Table presents details of the experimental hut studies evaluating different numbers of holes and displays blood feeding, mortality and exophily rates. Port and Boreham (1982) evaluated the protection afforded by untreated nets owned by the men who were employed to sleep in the experimental huts in The Gambia. They measured the physical integrity of nets by calculating the sum of the circumference of all holes and the lengths of any splits, which ranged from $0-195 \mathrm{~cm}$. Interestingly, the proportion of Anopheles gambiae s.s. feeding on volunteers using nets with few holes was not very different than those where unholed nets were used; indeed, the lowest proportion of feeding occurred on a volunteer under a net with nets with a hole measure of $16 \mathrm{~cm}$, though the relative attractiveness of the sleepers may have been important. Similarly, blood feeding on volunteers using nets with high hole measures was similar to blood feeding on volunteers not using nets.

Irish et al. (2008) and Ngufor et al. (2011) both evaluated mosquito nets with different numbers and sizes of holes in southern Benin. In both studies, nets with six holes of $4 \times 4 \mathrm{~cm}$ [now a standard for WHO Pesticide Evaluation Scheme (WHOPES) (WHO 2013)] were compared with nets with 80 holes of $2 \times 2 \mathrm{~cm}$ which had been used in previous studies (Asidi et al. 2004, N'Guessan et al. 2007). Six holes of $4 \times 4 \mathrm{~cm}$ is equivalent to $96 \mathrm{~cm}^{2}$ of holes $[96 \mathrm{~cm}$ of the Port and Boreham (1982) measure], while eighty $2 \times 2 \mathrm{~cm}$ is equivalent to $320 \mathrm{~cm}^{2}$ (640 $\mathrm{cm}$ of the hole measure). Irish et al. (2008), working in Ladji, near Cotonou, used alphacypermethrin-treated nets (Fendona $6 \mathrm{SC}$ ), conventionally treated at a dose of $40 \mathrm{mg} / \mathrm{m}^{2}$, while Ngufor et al. (2011), working in Akron, near Porto-Novo, used a long-lasting insecticidal net with a target dose of $55 \mathrm{mg} / \mathrm{m}^{2}$ of deltamethrin. Both studies observed significantly higher rates 
of Culex quinquefasciatus blood feeding with 80 holes compared with six holes, for both treated and untreated nets (in Ladji, the difference was not significant for untreated nets). However, despite increased blood feeding, the mortality rates for $C x$. quinquefasciatus was not significantly different between nets with different numbers of holes and was significantly higher with treated nets. In both studies, mortality was low, due to pyrethroid resistance in Cx. quinquefasciatus in southern Benin (Corbel et al. 2007). In the study in Akron, similar patterns were evident for An. gambiae (increased blood feeding with more holes, no difference in mortality), however, mortality of the pyrethroid-resistant An. gambiae (Yadouleton et al. 2010) was $37-50 \%$, slightly higher than with $C x$. quinquefasciatus (13-15\%).

Malima et al. (2008) reported greater blood feeding and less mortality of pyrethroid-susceptible An. gambi$a e$ and funestus under a seven-year old Olyset net (originally $2 \%$ permethrin $\mathrm{w} / \mathrm{w}$ ), which had developed holes (the size and number of holes is not reported), than under an Olyset which had six $4 \times 4 \mathrm{~cm}$ holes cut into it. The seven-year old Olyset still had some killing effect, as demonstrated in bioassays, but as the insecticidal effects were significantly less than the new Olyset, it is difficult to separate the effects due to reduced insecticide and those due to the holes in the net.

Studies with holed and intact nets - In experimental hut studies comparing results from holed and unholed nets, a general pattern emerged with increased blood feeding in huts with holed nets, as might be expected. As shown in Fig. 1, the majority of trials collected more blood-fed mosquitoes from huts where holed mosquito nets were used. However, the difference in blood feeding was coupled with very small or no difference in rates of mortality of mosquitoes exposed to treated nets with or without holes (Lines et al. 1987, Gokool et al. 1992, Curtis 1995, Irish et al. 2008). The minimal effect of holes in nets on the mortality of mosquitoes in experimental huts can be seen in Fig. 2. This pattern was found with several insecticide/species combinations. Note that certain references found by the literature search are not included in Figs 1, 2, due to data presented in other publications, insufficient information, or holed and unholed nets being tested in separate locations or at different times (Port \& Boreham 1982, Pan et al. 1995, Curtis et al. 1996, Miller 1999, Darriet et al. 2000).

However, there were several exceptions to this pattern, which occurred when intact nets were ripped during a study, when nets were untreated, or when nets were treated with a non-pyrethroid insecticide. Darriet et al. (1984), in one of the first experimental hut study on pyrethroid-impregnated bednets, compared bednets treated with permethrin at a concentration of $80 \mathrm{mg} / \mathrm{m}^{2}$ with untreated nets, with both types of nets holed for approximately $5 \%$ of the total area of the net $\left(0.5 \mathrm{~m}^{2}\right.$ for one-person nets and $0.675 \mathrm{~m}^{2}$ for two-person nets). For both treated and untreated nets, the blood feeding rates of An. gambiae and An. funestus were quite high (75-97\%) and slightly higher in holed nets than unholed nets (never more than $6 \%$ higher), with the exception of

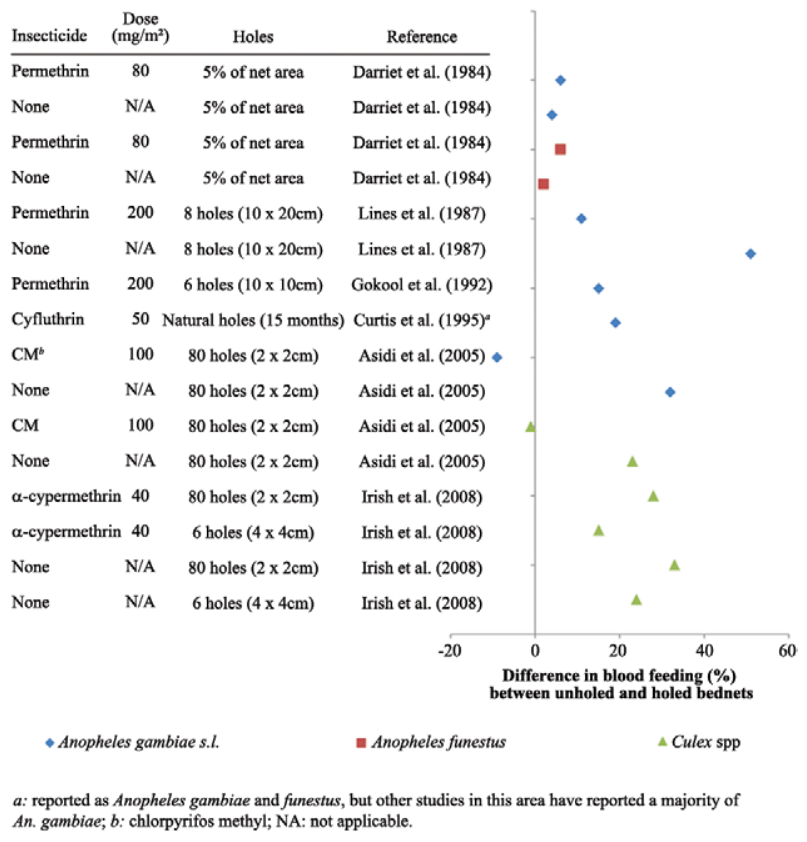

Fig. 1: differences in blood feeding between mosquitoes collected in experimental huts where holed and unholed nets were compared (percent blood-fed with intact nets - percent blood-fed with holed nets).

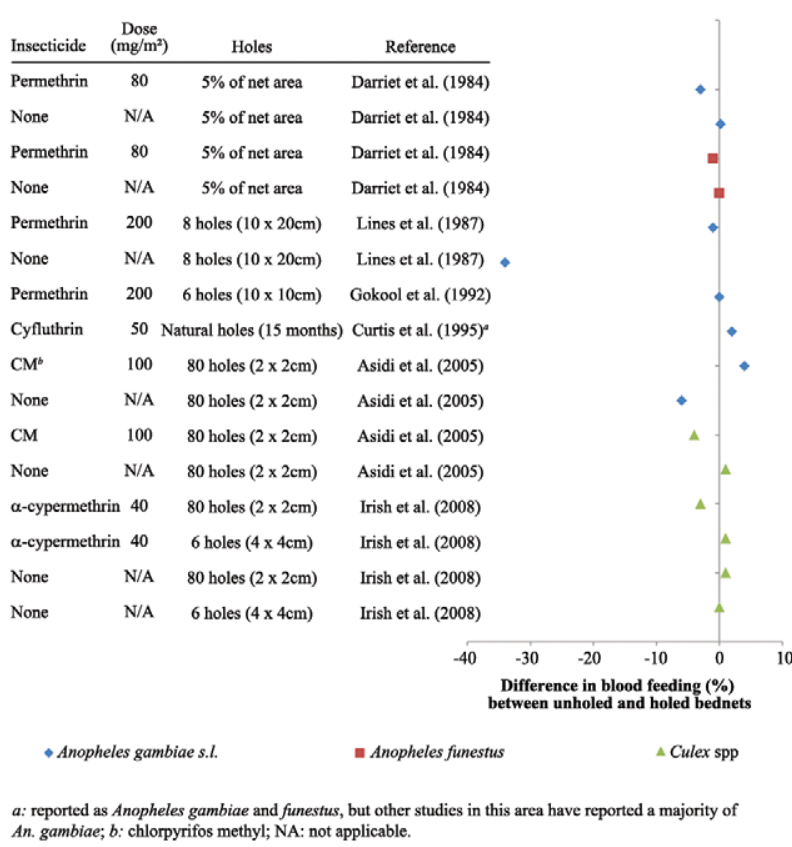

Fig. 2: differences in mortality between mosquitoes collected in experimental huts where holed and unholed nets were compared (percent dead with intact nets - percent dead with holed nets).

An. funestus where the blood feeding rate was $85 \%$ under holed nets and $91 \%$ under intact nets. The authors explained these small differences by noting that the nets that began the trial as intact nets were quickly torn by the users over the course of the trial. It should also be noted that the dose of permethrin on the nets $(80 \mathrm{mg} /$ 
$\mathrm{m}^{2}$ ) was lower than currently recommended levels of $200-500 \mathrm{mg} / \mathrm{m}^{2}$ (WHOPES 2007). There was almost no mortality of An. gambiae in the huts with untreated nets, while the mortality between huts with holed and unholed nets was nearly the same for one-person nets ( $18 \%$ under intact nets, 22\% under holed nets). Curtis et al. (1996) present a large amount of experimental hut data on mosquito nets with different insecticides, fabrics, months of prior use and number of washes. They found fairly low blood feeding rates by Anopheles mosquitoes on sleepers under nets treated with permethrin $(200-500 \mathrm{mg} /$ $\left.\mathrm{m}^{2}\right)$, deltamethrin $\left(3-25 \mathrm{mg} / \mathrm{m}^{2}\right)$ and lambdacyhalothrin $\left(3-15 \mathrm{mg} / \mathrm{m}^{2}\right)$, with geometric means of less than $45 \%$ for all three treatments, holed or unholed. When comparing new nets with the same insecticide dose and fabric, nets with six holes $(4 \times 4 \mathrm{~cm})$ had between three and nine times more blood feeding than nets without holes. Mortality was higher in nets with holes, except for permethrin $\left(500 \mathrm{mg} / \mathrm{m}^{2}\right)$, which had higher mortality under intact nets (38\%) compared with holed nets (29\%). It is difficult to compare these results as it seems they were not conducted at the same time (hut work was done over 4 years). Asidi et al. (2005), working in Cote d'Ivoire, found only small differences in blood feeding or mortality when holes ( 80 holes of $2 \times 2 \mathrm{~cm}$ ) were added to nets treated with chlorpyrifos methyl $\left(100 \mathrm{mg} / \mathrm{m}^{2}\right)$. However, they found increases in blood feeding when holes were added to untreated nets.

\section{DISCUSSION}

As shown in studies with different sizes or numbers of holes, or in studies with and without holes, the general and unsurprising, observation is that with increased holes there is increased blood feeding by mosquitoes in experimental huts. Before continuing, it should be noted that the presence of blood-fed mosquitoes in an experimental hut do not mean that the mosquitoes have fed on the person inside the hut (Kitau et al. 2014).There are several factors that influence a mosquito's ability to feed on a human protected by a net, including the presence of holes in the net, the resistance of the mosquito to the insecticide on the net and the proximity of the human to the net (determined by the size of the net, the number of people inside the net, the sleeping location of the human, etc.), which could allow feeding through the net. Another factor is the effect of the location of holes on the net. No record of the roofs of nets being holed was noted, but in recent behavioural studies, it was found that much of the activity of An. gambiae occurs at the roof (Lynd \& McCall 2013, Sutcliffe \& Yin 2014). It may be that holes in the roof are more important than holes on the sides of a net, which would have obvious importance for net manufacturers.

Despite the trend for increased feeding on people under nets when holes were present, a small number of holes did not render a net completely ineffective. Indeed, in Phase II tests of LLINs, nets are holed (ie., 6 holes of 4 $\mathrm{x} 4 \mathrm{~cm}$ ) to replicate the conditions of used nets. All currently approved LLINs are able to provide over $70 \%$ blood feeding inhibition even with holes in the net, sometimes maintaining this effect even after 20 washes (WHOPES 2004, 2008, 2009a, b, 2011, 2012, Pennetier et al. 2013).
The second characteristic that was evaluated in this review was the mortality of mosquitoes in relation to holes in nets. It was found that often the killing effect of a net was not reduced by perforation of the net. Indeed, with the exception of one result in an untreated net (Lines et al. 1987), the mortality of An. gambiae s.l. in huts with holed nets was never more than $6 \%$ different from that of huts with intact nets (Fig. 2). One potential reason for this lack of difference is that An. gambiae s.l. succeeding in entering a net to feed must also exit the net. Some mosquitoes do not leave the bednet and remain inside the bednet until they are collected or die. Lines et al. (1985) reported mortality and blood feeding results for mosquitoes collected under treated and untreated nets as well as the results for mosquitoes collected in other parts of the hut. They found that for the untreated nets, mosquitoes collected under the net had higher blood feeding than mosquitoes collected elsewhere, both when nets were holed and unholed. The mortality of mosquitoes collected inside the untreated nets was less than mosquitoes collected elsewhere, both for holed nets and unholed nets. However, although the addition of $200 \mathrm{mg} / \mathrm{m}^{2}$ of permethrin did not change the finding of a higher proportion of blood-fed mosquitoes under the net, the mortality of mosquitoes under nets was greater than that of those outside the net. Blood- fed mosquitoes may have different levels of insecticide susceptibility and this effect is not fully understood, as certain factors such as the age of the mosquito or the time after blood feeding can affect susceptibility (Davidson 1958, Rajatileka et al. 2011). It should be noted that defensive movements of humans sleeping under a net may also result in some increased mortality.

Therefore, a holed net might not provide the same personal protection as an intact net, but may continue to kill mosquitoes. Killing mosquitoes, even in the absence of personal protection, should be considered when making decisions about vector control (Killeen et al. 2011).

An important effect of holes which cannot be measured in experimental huts is the impact that holes have on the use of the net. In some situations, nets become so torn or holed that homeowners no longer consider them useful (Maxwell et al. 2002). Net use has also been strongly correlated with net condition (Kilian et al. 2011).

The importance of holes in nets in experimental huts is of obvious importance for entomologists, but the larger question is what effect do holes have on the biting and malaria transmission in the field. Rehman et al. (2011) found reduced odds of malaria when nets had no or few holes. However, as shown by successful WHOPES trials, the effectiveness of a net is not determined uniquely by its physical integrity. A net's success is determined by three factors: the insecticidal activity on mosquito vectors, the physical separation a net provides between mosquito vectors and the human and the way in which the net is used (time of use, frequency of use, factors such as tucking in the net, etc.). A "failed net" would be one that no longer provides any protection to the sleeper or any insecticidal effect which might provide protection to someone else.

Of major concern to national malaria control programs is the timing of net distribution campaigns. It 
is clear that nets should be distributed and sensitisation activities undertaken before all $100 \%$ nets fail, but as shown by Malima et al. (2008), some nets can have insecticidal effects at seven years. It seems likely that homeowners would discard a net before the insecticide on the net is reduced to a level where it has no effect on mosquitoes and the physical structure of a net provides no protection. The distribution of mosquito nets and accompanying sensitisation activities should occur at a rate to reduce transmission of malaria. It seems reasonable to create a matrix of factors which influence the success of nets to determine when these activities take place and it is very likely that these activities will have to be conducted at the national scale, if not at a more detailed level, to ensure success of malaria control programs based on distribution of LLINs.

\section{ACKNOWLEDGEMENTS}

Olivier Briët, David Hoel, Keren Landman, Graham White, Jacklyn Wong and John Gimnig are thanked for their input on this review.

\section{REFERENCES}

Asidi A, N'Guessan R, Akogbeto M, Curtis C, Rowland M 2012. Loss of household protection from use of insecticide-treated nets against pyrethroid-resistant mosquitoes, Benin. Emerg Infect Dis 18: 1101-1106.

Asidi AN, N'Guessan R, Hutchinson RA, Traoré-Lamizana M, Carnevale P, Curtis CF 2004. Experimental hut comparisons of nets treated with carbamate or pyrethroid insecticides, washed or unwashed, against pyrethroid-resistant mosquitoes. Med Vet Entomol 18: 134-140.

Asidi AN, N'Guessan R, Koffi AA, Curtis CF, Hougard JM, Chandre F, Corbel V, Darriet F, Zaim M, Rowland MW 2005. Experimental hut evaluation of bednets treated with an organophosphate (chlorpyrifos-methyl) or a pyrethroid (lambdacyhalothrin) alone and in combination against insecticide-resistant Anopheles gambiae and Culex quinquefasciatus mosquitoes. Malar J 4: 25.

Batisso E, Habte T, Tesfaye G, Getachew D, Tekalegne A, Kilian A, Mpeka B, Lynch C 2012. A stitch in time: a cross-sectional survey looking at long lasting insecticide-treated bed net ownership, utilization and attrition in SNNPR, Ethiopia. Malar J 11: 183.

Corbel V, N'Guessan R 2013. Distribution, mechanisms, impact and management of insecticide resistance in malaria vectors: a pragmatic review. In S Manguin, Anopheles mosquitoes - New insights into malaria vectors, InTech, Rijeka, p. 579-633.

Corbel V, N'Guessan R, Brengues C, Chandre F, Djogbenou L, Martin T, Akogbeto M, Hougard JM, Rowland M 2007. Multiple insecticide resistance mechanism in Anopheles gambiae and Culex quinquefasciatus from Benin, West Africa. Acta Trop 101: 207-216.

Curtis CF 1995. Impregnated bednets for malaria vector control. Bayer Public Health 12: 24-29.

Curtis CF, Myamba J, Wilkes TJ 1996. Comparison of different insecticides and fabrics for anti-mosquito bednets and curtains. Med Vet Entomol 10: 1-11.

Darriet F, N'Guessan R, Carnevale P 2000. Evaluations en cases-pièges de l'effet protecteur de moustiquaires non imprégnées d'insecticide dans la prévention des piqûres d'Anopheles gambiae s.s. Cahiers d'Etudes et de Recherches Francophones/Santé 10: 413-417.
Darriet F, N'Guessan R, Koffi A, Konan LY, Doannio IMC, Chandre F, Carnevale P 2000. Impact de la résistance aux pyréthrinoïdes sur l'efficacité des moustiquaires imprégnées dans la prévention du paludisme: résultats des essais en cases expérimentales avec deltaméthrine SC. Bull Soc Pathol Exot 95: 131-134.

Darriet F, Vincent R, Vien NT, Carnevale P, World Health Organization 1984. Evaluation de l'efficacité sur les vecteurs du paludisme de la perméthrine en imprégnation sur des moustiquaires intactes et trouées, Organisation Mondiale de la Santé, Geneve, 35 pp.

Davidson G 1958. Studies on insecticide resistance in anopheline mosquitos. Bull World Health Organ 18: 579-621.

Gokool S, Smith DF, Curtis CF 1992. The use of PCR to help quantify the protection provided by impregnated bednets. Parasitol Today 8: $347-350$.

Henry M-C, Assi S-B, Rogier C, Dossou-Yovo J, Chandre F, Guillet P, Carnevale P 2005. Protective efficacy of lambdacyhalothrin treated nets in Anopheles gambiae pyrethroid resistant areas of Côte d'Ivoire. Am J Trop Med Hyg 73: 859-864.

Irish SR, N'Guessan R, Boko PM, Metonnou C, Odjo A, Akogbeto M, Rowland M 2008. Loss of protection with insecticide-treated nets against pyrethroid-resistant Culex quinquefasciatus mosquitoes once nets become holed: an experimental hut study. Parasit Vectors 1: 17.

Kilian A, Byamukama W, Pigeon O, Gimnig J, Atieli F, Koekemoer L, Protopopoff N 2011. Evidence for a useful life of more than three years for a polyester-based long-lasting insecticidal mosquito net in western Uganda. Malar J 10: 299.

Killeen GF, Chitnis N, Moore SJ, Okumu FO 2011. Target product profile choices for intra-domiciliary malaria vector control pesticide products: repel or kill? Malar J 10: 20.

Kitau J, Oxborough R, Kaye A, Chen-Hussey V, Isaacs E, Matowo J, Kaur H, Magesa SM, Mosha F, Rowland M, Logan J 2014. Laboratory and experimental hut evaluation of a long-lasting insecticide treated blanket for protection against mosquitoes. Parasit Vectors 7: 129.

Lines JD, Curtis CF, Myamba J, Njau R, World Health Organization/ Division of Vector Biology and Control 1985. Tests of repellent or insecticide impregnated curtains, bednets and anklets against malaria vectors in Tanzania, WHO, Geneva, $16 \mathrm{pp}$.

Lines JD, Myamba J, Curtis CF 1987. Experimental hut trials of permethrin-impregnated mosquito nets and eave curtains against malaria vectors in Tanzania. Med Vet Entomol 1: 37-51.

Lynd A, McCall PJ 2013. Clustering of host-seeking activity of Anopheles gambiae mosquitoes at the top surface of a humanbaited bed net. Malar J 12: 267.

Malima RC, Magesa SM, Tungu PK, Mwingira V, Magogo FS, Sudi W, Mosha FW, Curtis CF, Maxwell C, Rowland M 2008. An experimental hut evaluation of Olyset nets against anopheline mosquitoes after seven years' use in Tanzanian villages. Malar J 7: 38 .

Maxwell CA, Msuya E, Sudi M, Njunwa KJ, Carneiro IA, Curtis CF 2002. Effect of community-wide use of insecticide-treated nets for 3-4 years on malaria morbidity in Tanzania. Trop Med Int Health 7: 1003-1008.

Mejía P, Teklehaimanot HD, Tesfaye Y, Teklehaimanot A 2013. Physical condition of Olyset ${ }^{\circledR}$ nets after five years of utilization in rural western Kenya. Malar J 12: 158.

Miller JE, Buriyo A, Karugila A, Lines JD 1999. A new strategy for treating nets. Part 1: formulation and dosage. Trop Med Int Health 4: $160-166$. 
N'Guessan R, Corbel V, Akogbéto M, Rowland M 2007. Reduced efficacy of insecticide-treated nets and indoor residual spraying for malaria control in pyrethroid resistance area, Benin. Emerg Infect Dis 13: 199-206.

Ngufor C, N'Guessan R, Boko P, Odjo A, Virginou E, Asidi A, Akogbeto M, Rowland M 2011. Combining indoor residual spraying with chlorfenapyr and long-lasting insecticidal bed nets for improved control of pyrethroid-resistant Anopheles gambiae: an experimental hut trial in Benin. Malar J 10: 343.

Pan B, Li ZZ, Huang QL 1995. Studies and application of pyrethroid treated bednets for control of malaria vectors in PR China. Chin $J$ Parasitic Dis Contr 8: 54-58.

Pennetier C, Bouraima A, Chandre F, Piameu M, Etang J, Rossignol M, Sidick I, Zogo B, Lacroix M-N, Yadav R, Pigeon O, Corbel V 2013. Efficacy of Olyset ${ }^{\mathbb{E}}$ Plus, a new long-lasting insecticidal net incorporating permethrin and piperonyl-butoxide against multiresistant malaria vectors. PLOS ONE 8: e75134.

Port GR, Boreham PFL 1982. The effect of bed nets on feeding by Anopheles gambiae Giles (Diptera: Culicidae). Bull Entomol Res 72: $483-488$

Rajatileka S, Burhani J, Ranson H 2011. Mosquito age and susceptibility to insecticides. Trans R Soc Trop Med Hyg 105: 247-253.

Ranson H, N'Guessan R, Lines J, Moiroux N, Nkuni Z, Corbel V 2011. Pyrethroid resistance in African anopheline mosquitoes: what are the implications for malaria control? Trends Parasitol 27: 91-98.

Rehman AM, Coleman M, Schwabe C, Baltazar G, Matias A, Gomes IR, Yellott L, Aragon C, Nchama GN, Mzilahowa T, Rowland M, Kleinschmidt I 2011. How much does malaria vector control matter: the epidemiological impact of holed nets and inadequate indoor residual spraying. PLOS ONE 6: r19205.

Strode C, Donegan S, Garner P, Enayati AA, Hemingway J 2014. The impact of pyrethroid resistance on the efficacy of insecticidetreated bednets against African anopheline mosquitoes: systematic review and meta-analysis. PLoS Med 11: e1001619.
Sutcliffe JF, Yin S 2014. Behavioural responses of females of two anopheline mosquito species to human-occupied, insecticidetreated and untreated bed nets. Malar J 13: 294.

Tami A, Mubyazi G, Talbert A, Mshinda H, Duchon S, Lengeler C 2004. Evaluation of Olyset insecticide-treated nets distributed seven years previously in Tanzania. Malar J 3: 19.

van den Berg H, Kelly-Hope LA, Lindsay SW 2013. Malaria and lymphatic filariasis: the case for integrated vector management. Lancet Infect Dis 13: 89-94.

WHO - World Health Organization 2012. Global plan for insecticide resistance management in malaria vectors (GPIRM), WHO, Geneva, $132 \mathrm{pp}$.

WHO - World Health Organization 2013. Guidelines for laboratory and field-testing of long-lasting insecticidal nets, WHO, Geneva, $89 \mathrm{pp}$.

WHOPES - WHO Pesticide Evaluation Scheme 2004. Report of the Seventh WHOPES Working Group Meeting, WHO, Geneva, 78 pp.

WHOPES - WHO Pesticide Evaluation Scheme 2007. WHO recommended insecticide products treatment of mosquito nets for malaria vector control. Available from: who.int/whopes/en/.

WHOPES - WHO Pesticide Evaluation Scheme 2008. Report of the Eleventh WHOPES Working Group Meeting, WHO, Geneva, 105 pp.

WHOPES - WHO Pesticide Evaluation Scheme 2009a. Report of the Twelfth WHOPES Working Group Meeting, WHO, Geneva, 127 pp.

WHOPES - WHO Pesticide Evaluation Scheme 2009b. Report of the Thirteenth WHOPES Working Group Meeting, WHO, Geneva, $81 \mathrm{pp}$.

WHOPES - WHO Pesticide Evaluation Scheme 2011. Report of the Fourteenth WHOPES Working Group Meeting, WHO, Geneva, 86 pp.

WHOPES - WHO Pesticide Evaluation Scheme 2012. Report of the Fifteenth WHOPES Working Group Meeting, WHO, Geneva, 107 pp.

Yadouleton A, N'Guessan R, Allagbé H, Asidi A, Boko M, Osse R, Padonou G, Kindé G, Akogbéto M 2010. The impact of the expansion of urban vegetable farming on malaria transmission in major cities of Benin. Parasit Vectors 3: 118. 